\title{
Systematic review of pre-operative exercise in colorectal cancer patients
}

Catherine Boereboom ${ }^{1}$, Brett Doleman ${ }^{2}$, Jonathan N Lund ${ }^{3}$, John P Williams ${ }^{4}$

${ }^{1}$ Research Fellow in Surgery, University of Nottingham, Derby, UK

${ }^{2}$ Research Fellow in Anaesthesia, University of Nottingham, Derby, UK

${ }^{3}$ Associate Professor and Consultant Colorectal Surgeon, University of Nottingham, Royal Derby Hospital, UK

${ }^{4}$ Associate Professor and Consultant Anaesthetist, University of Nottingham, Royal Derby Hospital, UK

*Corresponding author

* Dr Brett Doleman

Division of Medical Sciences and Graduate Entry Medicine

University of Nottingham

Royal Derby Hospital

Uttoxeter Road

Derby

DE22 3DT

Telephone: 01332724641

Email: dr.doleman@gmail.com

\section{Acknowledgements}

The authors declare that they have no conflicts of interest. We would like to acknowledge the help of Suzanne Toft, the clinical librarian who conducted the electronic search strategy. 


\begin{abstract}
Purpose

The aim of this systematic review was to evaluate the evidence for exercise interventions prior to surgery for colorectal cancer resection. The evidence for use of exercise to improve physical fitness and surgical outcomes is as yet unknown.
\end{abstract}

\title{
Method
}

A systematic search was performed of MEDLINE, EMBASE, CINAHL, AMED and BNI databases for studies involving pre-operative exercise in colorectal cancer patients.

\section{Results}

Eight studies were included in the review. There is evidence that pre-operative exercise improves functional fitness, and to a lesser extent objectively measurable cardio-respiratory fitness prior to colorectal cancer resection. There is no clear evidence at present that this improvement in fitness translates into reduced peri-operative risk or improved post-operative outcomes. Current studies are limited by risk of bias.

\section{Conclusions}

This review highlights the common difficulty in transferring promising results in a research setting, into significant improvements in the clinical arena. Future research should focus on which type of exercise is most likely to maximise patient adherence and improvements in cardio-respiratory fitness. Ultimately, adequately powered, randomised controlled trials are needed to investigate if pre-operative exercise improves post-operative morbidity and mortality.

\section{Keywords}

Exercise; colorectal cancer; systematic review; mortality 


\section{Introduction}

Colorectal cancer is the third most common malignancy in the UK and represents a significant healthcare burden to society. There are many associations along the cancer pathway between physical fitness and cancer. A body of population-based, epidemiological research concludes that increases in exercise are associated with a reduction in the primary risk of developing colorectal cancer [1] and can improve survival after colorectal cancer treatment [2]. The benefits of physical activity to protect against the development of multiple cardio-respiratory and metabolic illnesses and their complications, as well as malignant disease, is also well documented and has led to the development of worldwide guidelines on exercise recommendations [3].

The role of exercise in colorectal cancer management has also been investigated in the management of post-treatment colorectal cancer survivors. However, despite the research literature indicating that exercise following cancer treatment confers a reduction in mortality [4], decreased risk of disease recurrence and improved quality of life [5], currently there is only limited evidence supporting the benefit of exercise during the surgical and oncological management of active colorectal cancer [6].

In the UK the time available to improve cardio-respiratory fitness pre-operatively is restricted by national cancer treatment targets, which stipulate that treatment for cancer must start within 31 days of the decision to treat [7]. The time pressure experienced in the UK and internationally may well contribute to the lack of quality research and subsequent evidence base supporting the use of exercise treatments in the period between decision to treat and surgery for colorectal cancer. Given however, that recent work has shown that those with improved cardio-respiratory fitness have lower rates of mortality and complications following colorectal cancer surgery [8,9], this time period provides an opportunity to attempt to improve cardio-respiratory fitness before surgery, with the possibility of reducing peri-operative risk and improving post-operative outcome.

In 2014, a meta-analysis evaluated the effect of exercise training in colorectal cancer patients [10] through all stages of the treatment pathway. This meta-analysis included studies published until the end of 2012 and found only three randomised controlled trials for inclusion, with all reporting effects of training only in patients who had completed colorectal cancer treatment. Since this review, there have been several investigations of exercise training programmes in the pre-operative cancer patient. Consequently there is a need to revisit the evidence supporting the use of pre-operative exercise interventions in this patient group.

Given emerging data regarding exercise prehabilitation and the lack of definitive, large-scale, high quality research in this area, this review aims to examine the current evidence base for pre-operative exercise in colorectal cancer patients. 


\section{Methods}

\section{Study design and participants}

This review was registered on the PROSPERO database prior to the literature search (registration number CRD42014015556). PRISMA guidelines for systematic reviews were followed [11]. Studies were included if they were randomised or cohort studies involving any type of exercise in the preoperative period in adults awaiting curative resection for their primary colorectal cancer. Studies with control groups who did no exercise and those with pre and post exercise intervention measurements but no control group were included. Exclusion criteria included studies investigating solely respiratory muscle training, post-operative exercise and studies investigating exercise prior to palliative surgery.

\section{Systematic literature search}

A clinical librarian performed the literature search. Articles were searched without language or date restriction (published up until 12th November 2014). MEDLINE, EMBASE, CINAHL, AMED and BNI databases were searched. The Cochrane Library was searched for evidence based reviews and Dynamed, PEMSoft and NICE Guidance for clinical guidelines. Clinicaltrials.gov website was searched for relevant unpublished studies. Reference lists of the identified primary studies (including previous review articles) were hand-searched for further studies.

Medical subject headings (MeSH) were used including the terms 'NEOPLASMS', 'COLORECTAL NEOPLASMS', 'COLORECTAL SURGERY', 'PREOPERATIVE CARE' and 'EXERCISE'. Freetext words included 'exercise' and 'pre AND operative'. Abstracts of identified studies were screened by two authors independently (CB and $\mathrm{BD})$. Full text versions of potentially eligible studies were retrieved. These were assessed independently by two study authors against the inclusion/exclusion criteria $(\mathrm{CB}$ and $\mathrm{BD})$ and agreement was reached by consensus.

\section{Outcomes}

Outcomes included post-operative mortality, post-operative length of hospital stay, post-operative complications, cardio-pulmonary exercise test markers of fitness, markers of functional fitness, quality of life measures, tumour recurrence, changes in tumour biology and biochemical inflammatory markers.

\section{Data extraction}

Data regarding study characteristics were extracted onto an electronic database. Risk of bias was assessed independently by two study authors (CB and BD) using the Newcastle Ottawa Quality Assessment scale for cohort studies [12] and the Cochrane tool for assessing risk of bias [13] for randomised controlled trials. Discrepancies were resolved by consensus. 


\section{Data synthesis}

Due to significant clinical heterogeneity in the type of exercise interventions, meta-analysis was deemed inappropriate. There was heterogeneity in the nature of the colorectal pathology, additional aspects of prehabilitation (eg. diet modification), control group interventions and length of exercise prehabilitation offered. 


\section{Results}

\section{Description of included and excluded studies}

Nine studies were identified and underwent full text review [14-22] (Figure 1). One hundred and twenty two studies were identified from the initial literature search and twenty-one from handsearching the study references and through other sources. No further studies were identified from searching clinical trial databases for unpublished studies.

One hundred and thirty four studies were excluded as they were duplicate publications or did not adhere to our inclusion criteria. Nine studies underwent full text review and one of these studies was excluded as it included non-colorectal cancer patients [14]. Therefore, eight studies were included in the qualitative synthesis.

Information on the characteristics of the included studies is shown in Table 1. The majority of the current work on exercise in pre-operative colorectal cancer patients has been undertaken by groups at McGill University in Canada and between Aintree and Southampton in the UK and have been published in the last 5 years. Mayo et al. [21] was a data re-analysis of Carli et al. [16]. Burke et al. [15] used a subsection of patients enrolled in the West study [22].

\section{Quality assessment of included studies}

All studies were at high risk of bias due to the impracticalities of blinding participants and exercise providers to the exercise intervention (Figure 2). Several studies blinded those interpreting the outcomes of the exercise intervention and thus reduced the risk of bias in this regard. Accepting the difficulties in blinding participants, five of the six studies assessed using the Cochrane tool for assessing risk of bias had one or more other domains assessed as at high risk of bias. Both West et al. [22] and Kim et al. [19] had significant baseline differences between the exercise and control groups. In several domains across the studies there was not enough methodological detail to assess risk of bias accurately. Gillis et al. [18] was at low risk of bias (accepting the lack of blinding of the participants).

\section{Description of interventions and compliance}

Gillis et al. [18] used a total-body exercise prescription, which consisted of 50 minutes of home-based exercise (unsupervised) for 3 days per week. Exercise involved any 20 minutes of aerobic activity that achieved a target heart rate and 20 minutes of resistance training (using major muscle groups). In addition to exercise, participants were given a dietary intervention, which included a protein supplement and a psychologist who instructed participants on relaxation techniques. Compliance with the intervention was $78 \%$. West et al. [22] used a supervised in-hospital intervention over a 6-week period with 3 sessions per week. The exercise consisted of 40 minutes on an electromagnetically 
braked bike with each participants exercise programme adjusted according to their CPET results. Compliance was high at 96\%. Burke et al. [15] used the same intervention as West et al. [22].

Li et al. [20] asked participants to walk or use an aerobic machine for 30 minutes, 3 times per week at half the calculated maximal heart rate in addition to resistance exercises. Participants were also given dietary advice and given a protein supplement with a session from a psychologist to perform anxietyreducing techniques. Full compliance with the intervention was $45 \%$. Carli et al. [16] used two intervention groups with one group undergoing bike and strengthening training. Participants were instructed to exercise at $50 \%$ maximal heart rate increasing by $10 \%$ each week and weight/resistance training 3 times per week. The other group were encouraged to walk daily for 30 minutes and perform deep breathing exercises. Full compliance was only 16\% in the bike/strengthening group. Mayo et al. [21] used the same intervention as Carli et al. [16].

Kim et al. [19] used a four-week aerobic exercise programme which was customised for each participant based on heart rate reserve and rating of perceived exertion. Compliance was $74 \%$. Dronkers et al. [17] had participants exercise twice per week over a 2-4 week period in an outpatient department. Each session lasted 60 minutes, was supervised and involved a warm up, lower extremity resistance training, inspiratory muscle training, aerobic training, functional activities and a cool down. Participants also followed a home-based programme that involved walking or cycling for 30 minutes per day and inspiratory muscle training. Attendance at the supervised sessions was $97 \%$.

\section{Post-operative outcomes}

No studies reported post-operative mortality. However, five studies reported post-operative surgical complications as a secondary outcome. Carli et al. [16] recorded Clavien-Dindo grades of postoperative complications (22/56 patients in the bike/strength group and 18/54 patients in the walk/breath group; $\mathrm{p}=0.56$ ). Gillis et al. [18] reported no difference in 30-day complications between those exercising pre-operatively and those exercising post-operatively (12/38 patients in pre-operative exercise group and 17/39 in the post-operative exercise group, $\mathrm{p}=0.28$ ). Li et al. [20] also reported no difference in post-operative complications (15/42 of the pre-operative exercise group and 20/45 of the control group; $\mathrm{p}=0.67)$. Dronkers et al. [17] also reported similar findings of no significant differences in post-operative complications with pre-operative exercise $(9 / 22$ and $8 / 20$ in the control group experienced a post-operative complication; $\mathrm{p}=0.65$ ).

In terms of length of stay, Carli et al. [16] found a longer mean length of stay in the bike/strengthening group (11.9 days and 6.6 days in the walk/breathing group). Dronkers et al. [17] found a reduction in length of stay in the pre-operative exercise group; however, this was not statistically significant (16.2 days and 21.6 days in the control group; $\mathrm{p}=0.31$ ). Similarly, Gillis et al. [18] found no difference in median length of stay in the pre-operative exercise group (4 and 4 days in the post-operative exercise 
group; $\mathrm{p}=0.81$ ). In addition, Li et al. [20] found no difference in median length of stay (4 and 4 days; $\mathrm{p}=0.71)$.

\section{Functional outcomes}

The primary end point of five studies was improvement in functional exercise capacity based on the 6minute walk test (6MWT). Gillis et al. [18] demonstrated a $25.2 \mathrm{~m}$ increase in 6MWT in their prehabilitation group compared to a $16.4 \mathrm{~m}$ decrease in those who did no pre-operative exercise $(p<0.001)$. Interestingly, at 8 weeks post-surgery, on average, the prehabilitation group had recovered to their baseline walking times and those who did no pre-operative exercise remained below baseline. Li et al. [20] also showed a significant improvement of 42m in 6MWT with prehabilitation and again this improvement in functional fitness over the control group persisted at $4(\mathrm{p}=0.01)$ and 8 weeks postoperatively $(\mathrm{p}<0.01)$.

Two studies did not show any improvement in functional fitness with prehabilitation. Kim et al. [19] showed no difference between their prehabilitation and control group with respect to improvement in $6 \mathrm{MWT}$, the groups improved by $31 \mathrm{~m}$ and $27 \mathrm{~m}$ respectively ( $\mathrm{p}$ value not given). Carli et al. [16] found the bike/strength group had a decrease in $6 \mathrm{MWT}$ of $10.6 \mathrm{~m}(\mathrm{p}=0.148)$ and the walk/breath group an increase of $8.7 \mathrm{~m}$ ( $\mathrm{p}=0.203)$, neither of these changes was significant. From the same data, Mayo et al. [21] looked at the group who completed the prehabilitation phase of the study (52\% bike/strength training and $48 \%$ walk/breath training). These data showed that $33 \%$ of the prehabilitation group improved their $6 \mathrm{MWT}$ regardless of their exercise regime, $38 \%$ did not change and $29 \%$ decreased their $6 \mathrm{MWT}$. Unsurprisingly, those who deteriorated in the prehabilitation phase had significantly lower post-operative 6MWT compared to their baseline.

\section{Cardio-respiratory physiology outcomes}

Two studies reported cardio-pulmonary exercise test variables eg. sub-maximal oxygen consumption $\left(\mathrm{VO}_{2}\right)$, anaerobic threshold (AT) and peak oxygen consumption $\left(\mathrm{VO}_{2}\right.$ peak) as their primary outcome. Kim et al. [19] reported a significant reduction of $2 \mathrm{ml} / \mathrm{kg} / \mathrm{min}(13 \%, \mathrm{p}>0.05)$ in submaximal oxygen consumption at a given submaximal workload after prehabilitation using a home based cycling exercise programme in less than 4 weeks. This was explained by an improvement in physiological efficiency at submaximal workloads following training, giving an improved physiological reserve. West et al. [22] evaluated patients undergoing neoadjuvant chemo-radiotherapy (NACRT) for locally advanced rectal tumours and showed a significant decrease in AT $(-1.91 \mathrm{ml} / \mathrm{kg} / \mathrm{min}, \mathrm{p}<0.001)$ and $\mathrm{VO}_{2}$ peak $(-$ $2.52 \mathrm{ml} / \mathrm{kg} / \mathrm{min}, \mathrm{p}<0.001)$. The exercise group then regained their cardio-pulmonary fitness over a 6 week exercise programme (prior to surgery) (AT $+2.12 \mathrm{ml} / \mathrm{kg} / \mathrm{min}, \mathrm{p}<0.001$ ) whilst the control group continued to decline (AT $-0.65 \mathrm{ml} / \mathrm{kg} / \mathrm{min}, \mathrm{p}=0.204$ ). This difference between the groups increased over the following 8 weeks (prior to surgery). 
Dronkers et al. [17] used Physical Work Capacity 170 to derive maximum aerobic capacity $\left(\mathrm{VO}_{2} \mathrm{max}\right)$ from power output at known heart rates during exercise. They found no change in this measurement following exercise training with a pre-operative $\mathrm{VO}_{2} \max$ of $27.6 \mathrm{ml} / \mathrm{kg} / \mathrm{min}$ and $32.9 \mathrm{ml} / \mathrm{kg} / \mathrm{min}$ in the intervention and control groups respectively $(\mathrm{p}=0.16)$.

\section{Quality of life outcomes}

Burke et al. [15] reported quality of life measures as their primary outcome showing qualitative improvements in sense of vitality, positive attitude, social connections and sense of purpose following pre-operative exercise training. Hermeneutic (the theory of text/interview interpretation) phenomenological methodology was used in this study to allow focus on patient's personal accounts of the study intervention rather than generic quality of life questionnaires.

Four studies [16,18,20,21] also reported quality of life outcome measures. Gillis et al. [18] found no difference between the prehabilitation and control group in any domains of the Short Form 36 Health Survey (SF-36) (73.5 and 72.6 respectively; adjusted $\mathrm{p}=0.47$ ) or Hospital Anxiety and Depression Scale (HADS) (in the anxiety domain before surgery, the prehabilitation group and control group scored 5.6 and 5.9 respectively; $\mathrm{p}=0.33$ ). The exercise group had higher SF-36 scores compared with the control group in Li et al. [20], although this difference was apparent at baseline and did not increase with exercise intervention, eg. in the general health domain before surgery, the prehabilitation and control groups scored 75 and 69 respectively $(\mathrm{p}=0.16)$.

Mayo et al. [21] noted that patients who had an improved 6MWT with pre-operative exercise also reported significant improvements in mental health, vitality and self-perceived health. Carli et al. [16] showed a significant reduction in HADS depression scores between baseline and surgery in those undergoing bike/strength training $(4.0$ to $3.2 ; \mathrm{p}=0.05)$ but not in those in the walk/breath group (3.6 to $3.4 ; \mathrm{p}=0.7)$.

\section{Other outcomes}

There was no investigation into colorectal cancer recurrence in the included studies. Tumour biology in response to pre-operative exercise was also not investigated. 


\section{Discussion}

This systematic review complements other recently published, more general review papers and metaanalyses on peri-operative exercise by focusing solely on pre-operative exercise in colorectal cancer patients. The current published evidence is mainly limited to work from three centres. Despite the heterogeneous nature of the exercise interventions used in the included studies, this review demonstrates that it is possible to improve the functional fitness of colorectal cancer patients preoperatively, with an increase in $6 \mathrm{MWT}$ of between $4 \mathrm{~m}$ and $42 \mathrm{~m}$ in intervention groups compared to controls.

The evidence for the feasibility of objectively improving cardio-respiratory fitness (as shown by increased $\mathrm{VO}_{2}$ peak or $\mathrm{AT}$ ) using exercise prior to surgery is thus far limited. The recently published study by West et al. [22] provides the best available current evidence to support the hypothesis that exercise training can improve objective measures of cardio-respiratory performance. However, their intervention took place over a 6-week period and had significant risk of bias due to lack of adequate randomisation resulting in imbalances in baseline characteristics.

From a clinical perspective, there is no evidence that improvements in physical performance translate into an improvement in post-operative outcomes. However, we believe that current studies are underpowered to adequately detect differences in this outcome. In order to demonstrate a statistically significant reduction of $10 \%$ in the absolute incidence of post-operative complications, studies would need to recruit around 400 participants with an alpha of 0.05 and a power of 0.80 . These data currently do not exist. Moreover, no study reported post-operative mortality as an outcome. Similarly, there is limited evidence to show improvements in quality of life measures following pre-operative exercise in colorectal cancer patients.

These findings are in line with a recent meta-analysis [10], which found evidence of short-term improvements in physical fitness but no associated evidence for improved survival. This meta-analysis included multiple cancer types and exercise interventions at many stages of the treatment pathway. Another review published a year before [23] evaluated exercise prior to surgery and found evidence that pre-operative aerobic exercise training was feasible, safe and improved one measure of physical fitness. There was data from only one study to show reduced length of post-operative stay. In contrast to these, a review published in the same year [24] evaluated exercise prehabilitation prior to a variety of surgical procedures including orthopaedic joint replacements, cardiac surgery and resections for intra-abdominal and thoracic malignancies. They concluded that there was evidence of reduced length of hospital stay and post-operative complications following pre-operative exercise interventions. However, poor methodology and high risk of bias was apparent in the studies they included in their review. 
Pre-operative exercise is limited by national targets requiring colorectal cancer surgery to be performed within one month [7]. This limits the time window in which to improve physical fitness. In addition, as these patients are often over 60 years old at diagnosis [25], this has implications for improving physical fitness prior to surgery as patient factors such as lower baseline functional status, co-morbidity (such as ischemic heart disease or osteoarthritis limiting exercise tolerance) and reduced muscle mass may affect their ability to undergo exercise programmes. Although recent research suggests there is consensus among surgeons that even patients at the extreme of age can have their physical fitness improved prior to surgery [26], it remains to be seen if such patients can benefit from such interventions. However, nearly all of the included studies in this review had a mean age above 60 years old which maintains the external validity of our findings. Unfortunately, due to clinical heterogeneity in the exercise programmes included in this review, it is difficult to suggest any specific exercise programme, which has implications for the design of future trials in the area. The lack of any objective data that indicates that pre-operative exercise improves clinical outcomes means our review cannot recommend that exercise interventions be introduced into routine clinical practice.

The studies included within this review are highly heterogeneous in terms of exercise interventions studied and compliance with these programmes. This limits the comparisons and makes recommending one programme over another problematic. Gillis et al. [18] used home-based interventions, which have advantages over supervised sessions, as they require less staff resources. However, compliance was only $78 \%$ in this study. Kim et al. [19] also used a home-based intervention and achieved similar compliance rates (74\%). Conversely, West et al. [22] and Dronkers et al. [17] used a supervised inhospital programme that both achieved high compliance rates of 96-97\%. Clearly, the effectiveness of any exercise programme is determined by both the effectiveness of the intervention in question and the compliance of participants undergoing the intervention. Therefore, future studies should investigate which forms of exercise interventions can deliver both improvements in physical fitness and achieve high rates of compliance.

There are several limitations with this review. The heterogeneous nature of the studies with regard to exercise interventions and outcome measures makes direct comparisons and meta-analysis problematic. Some of the domains used to assess internal validity are unclear from the study manuscripts. Therefore, some of the studies may be at a higher risk of bias than is apparent. Many of the underlying studies were at high risk for some domains, especially in regards to imbalances in baseline characteristics; this introduces selection bias, which may cloud interpretation of our results. Although only two of the studies we included were observational, interventional studies were mainly at high risk of bias, which would downgrade any evidence derived from these studies. When considering the higher quality evidence, only one randomized study [18] received low risk of bias for most domains (excluding blinding). This study showed improvements in functional outcomes but no differences in postoperative complications. Finally, additional interventions such as dietary supplements may confound interpreting direct benefits from exercise. 
In terms of future research studies, these should focus on what type of exercise programmes can achieve improvements in physical fitness within a suitable time period that corresponds to cancer treatment targets $(<4$ weeks in the UK). This will be the major limitation of introducing exercise programmes into clinical practice and future studies should ensure interventions are delivered within this period. Indeed, the study by West et al. [22] used a 6-week programme, which would be difficult to implement due to these national targets for treatment. In addition, such programmes need to be tolerable to the specific demographic of patients that undergo colorectal cancer surgery ( $>60$ years old) and whether such programmes are suitable and effective for older cohorts of patients ( $>75$ years old). As previously discussed, an important aspect of whether such programmes will be effective in clinical practice will be compliance rates and which interventions can achieve the highest rates of compliance. Results from our review suggest in-hospital programmes may achieve higher rates of compliance.

Ultimately, large randomised controlled trials are required to improve the internal validity of current findings. Although blinding of interventions would be difficult, adequate randomisation, allocation concealment and blinding of outcome assessment are possible in order to improve internal validity. In addition, studies should report both per-protocol results (to help assess how the exercise intervention can work when adhered to) and intention to treat analysis (as those who are non-compliant are more likely to have poor outcomes). Furthermore, they would need to be adequately powered to ensure they can detect differences in post-operative outcomes. Moreover, future clinical trials need to evaluate clinically relevant outcomes such as post-operative complications and mortality if pre-operative exercise interventions are to become standard clinical practice in patients undergoing colorectal cancer resection.

In conclusion, the current evidence on pre-operative exercise for colorectal cancer patients is limited by a lack of adequately powered, clinically relevant outcomes, heterogeneous interventions and risk of bias issues in the conduct of the studies published thus far. Whilst there is some evidence that preoperative exercise can improve both measures of physical fitness and functional fitness, there is no evidence that this in turn improves post-operative outcomes. 


\section{References}

[1] Harriss D, Cable N, George K, Reilly T, Renehan A, Haboubi N. (2007) Physical activity before and after diagnosis of colorectal cancer: disease risk, clinical outcomes, response pathways and biomarkers. Sports Med; 37:947-60.

[2] Haydon a MM, Macinnis RJ, English DR, Giles GG. (2006) Effect of physical activity and body size on survival after diagnosis with colorectal cancer. Gut; 55:62-67.

[3] WHO. Global recommendations on physical activity for health. WHO. 2010.

[4] Courneya KS, Booth CM, Gill S, O’Brien P, Vardy J, Friedenreich CM, et al. (2008) The Colon Health and Life-Long Exercise Change trial: a randomized trial of the National Cancer Institute of Canada Clinical Trials Group. Curr Oncol; 15:279-285.

[5] Denlinger CS, Engstrom PF. (2011) Colorectal cancer survivorship: movement matters. Cancer Prev Res; 4:502-511.

[6] Kuan-Yin L, Shiow-Ching S, Yeur-Hur L, Jin-Tung L, Jau-Yih T. (2014) Comparison of the effects of a supervised exercise program and usual care in patients with colorectal cancer undergoing chemotherapy. Cancer Nurs; 37:E21-9.

[7] National Cancer Action Team NCIN. Cancer waiting times: A Guide (Version 7).

[8] West M, Lythgoe D, Barben CP, Noble L, Kemp GJ, Jack S, et al. (2014) Cardiopulmonary exercise variables are associated with postoperative morbidity after major colonic surgery: a prospective blinded observational study. Br J Anaesth; 112:665-671.

[9] West M, Parry MG, Lythgoe D, Barben CP, Kemp GJ, Grocott MPW, et al. (2014)

Cardiopulmonary exercise testing for the prediction of morbidity risk after rectal cancer surgery. $\mathrm{Br} \mathrm{J}$ Surg; 101:1166-1172.

[10] Cramer H, Lauche R, Klose P, Dobos G, Langhorst J. (2014) A systematic review and metaanalysis of exercise interventions for colorectal cancer patients. Eur J Cancer Care (Engl); 23:3-14.

[11] Moher D, Liberati A, Tetzlaff J, Altman DG. (2009) Preferred reporting items for systematic reviews and meta-analyses. Ann Int Med; 151:264-269.

[12] Wells G, Shea B, Connell DO, Peterson J, Welch V, Losos M, et al. (2000) The Newcastle-Ottawa Scale (NOS) for assessing the quality of nonrandomised studies in meta-analyses.

[13] Higgins JPT, Altman DG, Gotzsche PC, Juni P, Moher D, Oxman a. D, et al. (2011) The Cochrane Collaboration's tool for assessing risk of bias in randomised trials. BMJ; 343:d5928-d5928.

[14] Timmerman H, de Groot JF, Hulzebos HJ, de Knikker R, Kerkkamp HEM, Meeteren V. (2011) Feasibility and prelimianary effectiveness of preoperative therapeutic exercise in patients with cancer: A pragmatic study. Physiother Theory Prac; 27:117-124.

[15] Burke SM, Brunet J, Sabiston CM, Jack S, Grocott MPW, West M. (2013) Patients' perceptions of quality of life during active treatment for locally advanced rectal cancer: the importance of preoperative exercise. Supportive Care Cancer; 21:3345-3353.

[16] Carli F, Charlebois P, Stein B, Feldman L, Zavorsky G, Kim DJ, et al. (2010) Randomized clinical trial of prehabilitation in colorectal surgery. Br J Surg; 97:1187-97.

[17] Dronkers JJ, Lamberts H, Reutelingsperger IMMD, Naber RH, Dronkers-Landman CM, Veldman a, et al. (2010) Preoperative therapeutic programme for elderly patients scheduled for elective abdominal oncological surgery: a randomized controlled pilot study. Clin Rehabil; 24:614-22. 
[18] Gillis C, Li C, Carli F. (2014) Prehabilitation versus rehabilitation: a randomized control trial in patients undergoing colorectal resection for cancer. Anesthesiology; 121: 937-947.

[19] Kim DJ, Mayo NE, Carli F, Montgomery DL, Zavorsky GS. (2009) Responsive measures to prehabilitation in patients undergoing bowel resection surgery. Tohoku J Exp Med; 217:109-115.

[20] Li C, Carli F, Lee L, Charlebois P, Stein B, Liberman AS, et al. (2013) Impact of a trimodal prehabilitation program on functional recovery after colorectal cancer surgery: a pilot study. Surg Endosc; 27:1072-1082.

[21] Mayo NE, Feldman L, Scott S, Zavorsky G, Kim DJ, Charlebois P, et al. (2011) Impact of preoperative change in physical function on postoperative recovery: argument supporting prehabilitation for colorectal surgery. Surgery; 150:505-514.

[22] West M, Loughney L, Lythgoe D, Barben CP, Sripadam R, Kemp GJ, et al. (2015) Effect of prehabilitation on objectively measured physical fitness after neoadjuvant treatment in preoperative rectal cancer patients: a blinded interventional pilot study. Br J Anaesth; 114:244-251.

[23] O’Doherty F, West M, Jack S, Grocott MPW. (2013) Preoperative aerobic exercise training in elective intra-cavity surgery: a systematic review. Br J Anaesth; 110:679-689.

[24] Santa Mina D, Clarke H, Ritvo P, Leung YW, Matthew a G, Katz J, et al. (2013) Effect of totalbody prehabilitation on postoperative outcomes: a systematic review and meta-analysis. Physiotherapy; 100: 196-207.

[25] Greenlee RT, Hill-Harmon MB, Murray T, Thun M. (2001). Cancer statistics, 2001. CA Cancer J Clin; 51: 15-36.

[26] Boereboom CL, Williams JP, Leighton P, Lund JN \& Exercise Prehabilitation in Colorectal Cancer Delphi Study Group. (2015). Forming a consensus opinion on exercise prehabilitation in elderly colorectal cancer patients: a Delphi study. Tech Coloproctol; 19: 347-354. 


\section{Legends to figures}

Fig 1: PRISMA flowchart of included and excluded studies

Fig 2: Risk of bias for included studies. Green indicates low risk, yellow unlcear risk and red high risk 